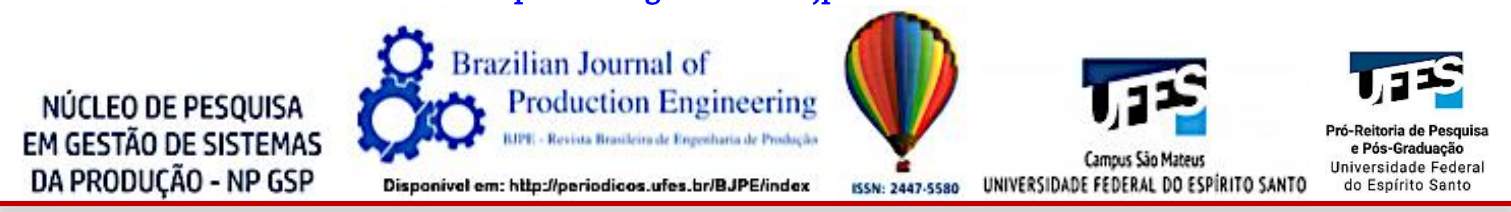

ARTIGO ORIGINAL

OPEN ACCESS

\title{
ANÁLISE DA REALIDADE AUMENTADA EM RELAÇÃO AO CONHECIMENTO DA POPULAÇÃO TEÓFILO-OTONENSE
}

\author{
ANALYSIS OF THE AUGMENTED REALITY WITH RESPECT TO THE KNOWLEDGE OF THE \\ TEÓFILO-OTONENSE POPULATION
}

\section{Daniela Faustin Carvalho Dias ${ }^{1 *}$, Américo Fernando de Souza Alves ${ }^{2}$, \& Luan Diego de Lima Pereira ${ }^{3}$ \\ 123 Instituto Federal do Norte de Minas Gerais, Campus Teófilo Otoni, IFNMG, Brasil. \\ 1* dfc1@aluno.ifnmg.edu.br ${ }^{2}$ americo.alves@ifnmg.edu.br ${ }^{3} \underline{\text { luan.pereira@ifnmg.edu.br }}$}

\section{ARTIGO INFO.}

\section{Recebido em:}

Aprovado em:

Disponibilizado em: 23.02.2022

\section{Palavras-chave:}

Realidade aumentada; tecnologia; integração.

KEYWORDS:

Augmented reality; technology; integration.

*Autor Correspondente: Dias, D. F. C.

\section{RESUMO}

A realidade aumentada é uma tecnologia presente no cotidiano das pessoas, ainda que elas a desconheçam. O ser humano tem dificuldade de acompanhar os avanços tecnológicos que têm surgido nas últimas décadas. Este trabalho propõe avaliar o grau de conhecimento da realidade aumentada na população teófilo-otonense a partir de dados coletados por meio de pesquisa elaborada no Google Forms contendo questionário baseado em survey exploratório. Para uma melhor amostra da pesquisa, foi utilizada a tabela de margem de erro de Arkin. A análise dos dados obtidos ocorreu por meio de gráficos e tabelas. Tais informações podem servir de subsídio para um planejamento de integração da população teófilootonense à Realidade Aumentada.

\begin{abstract}
Augmented reality is a technology present in people's daily lives, even if they do not know it. Human beings have difficulty keeping up with the technological advances that have emerged in recent decades. This paper proposes to evaluate the degree of knowledge of augmented reality in the teofilo-otonense population from data collected through a research elaborated in Google Forms containing a questionnaire based on an exploratory survey. For a better sample of the research, Arkin's margin of error table was used. The analysis of the obtained data occurred through graphs and tables. Such information may serve as a subsidy for the integration planning of the teofilo-otonense population to augmented reality.
\end{abstract}


Citação (APA): Dias, D. F. C., Alves, A. F., de S., \& Pereira, L. D., de L. (2022). Análise da realidade aumentada em relação ao conhecimento da população teófilo-otonense. Brazilian Journal of Production Engineering, 8(1), 107-117.

\section{INTRODUÇÃO}

Apesar de ser a $9^{\text {a }}$ (nona) maior economia do mundo, segundo o Índice Global de Inovação IGI (2020), o Brasil ocupa a 62a (sexagésima segunda) colocação no ranking de inovação que abrange 131 países. A classificação se torna incompatível se comparada ao ranking da economia.

Conforme afirma Robson Braga de Andrade, presidente da Confederação Nacional da Indústria (CNI), "o Brasil continua numa posição abaixo de seu potencial. Precisamos melhorar o financiamento à inovação, fortalecer parcerias entre governo, setor produtivo e academia, estruturar políticas de longo prazo e priorizar a formação de profissionais qualificados" (Abreu, 2020).

Sabe-se que o setor tecnológico evolui ininterruptamente. A realidade aumentada, doravante RA, está entre as inovações e se torna cada dia mais comum no cotidiano das pessoas, fato que traz a necessidade dos usuários se manterem atualizados sobre as novidades.

A RA permite ao usuário vivenciar o mundo real e o virtual ao mesmo tempo. Camargo et al. (2010) ressalta que a RA produz um único ambiente onde se tem a interação e a manipulação através das mãos. A origem da mesma se dá nos códigos Quick Response (QR). Como os códigos de barras foram se tornando limitados, foi necessário criar uma ferramenta que permitisse a inserção de mais informações levando a criação dos códigos duas dimensões (2D).

Milgran (1995, p. 283) diz que a RA "é a mistura de mundos reais e virtuais em algum ponto da realidade/virtualidade contínua, que conecta ambientes completamente reais a ambientes completamente virtuais".

Segundo Coifman (2020), a RA não é limitada às empresas tecnológicas, ela se aplica a variadas situações do dia a dia, se tornando tendência em diversos segmentos. Abaixo alguns exemplos:

-Jogos - um grande exemplo é o PokemónGo, o qual projeta os pokemóns que devem ser capturados. Através da câmera do celular, o usuário consegue visualizar os "monstrinhos" nos mais variados locais - praça, igreja, shopping, ruas e nos cômodos de sua casa;

-Medicina - através de óculos holográficos, o corpo humano, órgãos e sistemas são projetados em três dimensões (3D), auxiliando, dessa forma, o profissional a ter uma visão mais clara sobre a situação enfrentada;

-Arquitetura - permite o profissional criar projetos em 3D. Não somente as plantas, mas também os ambientes, de forma que possibilita ao cliente uma percepção de como ficará o resultado final.

Já Paiola (2019), cita as formas de aplicações da RA na indústria:

-Segurança do trabalho - a RA permite que os gestores monitorem os operadores, verificando se os mesmos estão utilizando os Equipamentos de Proteção Individual (EPI's) necessários, e se a condição que os operadores estão realizando as operações é segura;

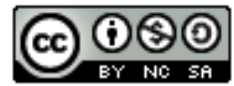


Citação (APA): Dias, D. F. C., Alves, A. F., de S., \& Pereira, L. D., de L. (2022). Análise da realidade aumentada em relação ao conhecimento da população teófilo-otonense. Brazilian Journal of Production Engineering, 8(1), 107-117.

- Treinamento e capacitações - permite a simulação das operações industriais, usando os óculos de realidade aumentada, tablets ou smartphones, aumentando a capacidade de aprendizagem;

-Manutenção Industrial - a RA pode facilitar a manutenção de equipamentos, por exemplo, quando o profissional tem acesso a uma animação virtual, na qual cada etapa necessária à manutenção de uma peça está especificada.

O grupo Iberdrola (2020), líder do setor energético global, descreve mais exemplos de aplicações da RA:

- Carros sob medida - onde os fabricantes permitem que os clientes visualizem como será o seu carro do futuro, adaptando os seus gostos;

- Lições que adquirem vida - permite que cadernos compostos de marcadores especiais, ofereçam aos alunos uma imagem 3D quando colocados em frente ao smartphone ou monitor do computador;

- Esportistas - permite que esportistas explorem os trajetos com projeção em 3D;

- Tradução - tradução instantânea através de uma foto tirada;

- Scanners faciais - reconhecimento da face, mostrando em quais redes sociais o usuário possui um perfil;

- Publicidade - permite que o usuário experimente uma roupa, por exemplo;

- O Universo mais perto - focando o celular no céu, o usuário pode identificar as estrelas, constelações, planetas e corpos celestes.

- NASA - óculos inteligentes que permitem aos astronautas receberem instruções para realizar manutenções durante as expedições.

Segundo a Agenzia Nazionale Stampa Associata - ANSA (2020), a Itália realizou a primeira cirurgia do mundo com realidade aumentada no dia 18 de fevereiro de 2020. O médico realizou a cirurgia facial utilizando uma espécie de óculos 3D, o Vostars, que mostra além dos objetos reais, objetos virtuais, que auxiliam em informações importantes para a realização da operação. Com isso, percebe-se que a realidade aumentada, além de algo para o lazer, é significativa para diversas operações, permitindo que várias áreas tenham seu trabalho otimizado e garantam maior taxa de sucesso.

Além da RA, existem a Realidade Virtual (RV) e a Realidade Estendida (XR, do inglês Extended Reality); é muito comum as pessoas confundirem as três tecnologias por serem semelhantes.

A interface em RV envolve um controle tridimensional altamente interativo de processos computacionais. $\mathrm{O}$ usuário entra no espaço virtual das aplicações e visualiza, manipula e explora os dados da aplicação em tempo real, usando seus sentidos, particularmente os movimentos naturais tridimensionais do corpo (Kirner, 1996). A RV insere o usuário em um ambiente artificial, ou seja, $100 \%$ virtual, que permite ao mesmo se movimentar em jogos, cenários e pontos turísticos. Têm-se como exemplos da RV jogos e imersões que utilizam os óculos VR. Diferente da RA, a RV cria um mundo novo no meio digital, sendo visto como uma nova realidade. 
Citação (APA): Dias, D. F. C., Alves, A. F., de S., \& Pereira, L. D., de L. (2022). Análise da realidade aumentada em relação ao conhecimento da população teófilo-otonense. Brazilian Journal of Production Engineering, 8(1), 107-117.

Já a XR envolve todo o conjunto de tecnologias relacionadas à produção de hardware, software e conteúdos nas áreas de RV e RA (Maciel, 2020), isto é, a junção das melhores partes da RV e da RA. Ela possibilita ao usuário outros tipos de interação além da visão, audição e tato, ou seja, o usuário consegue manipular e interagir com os elementos. Utilizamse simuladores e wearables, permitindo que as empresas criem experiências únicas aos clientes.

Nesse contexto, este estudo teve como objetivo identificar o grau de conhecimento da população em relação a RA, através de surveys exploratórios, e traçar o perfil da população quanto ao uso da RA, assim como propor ações para o uso da tecnologia no município.

\section{METODOLOGIA}

\section{Surveys}

Freitas et al. (2000) diz que a pesquisa do tipo Survey é utilizada quando se precisa obter dados sobre características, opiniões ou ações de um determinado grupo de indivíduos em relação a um determinado assunto, por meio de questionário.

Questionários baseados em surveys são utilizados para pesquisas nas diversas áreas da gestão de operações, levantando dados que permitem obter uma visão geral sobre o fenômeno em questão, de acordo com as variáveis pré-definidas pelo pesquisador, ou até mesmo concluir a respeito da relação de causa e efeito entre essas variáveis (Miguel, 2010). Ainda segundo o autor, surveys podem ser divididos em três tipos, conforme o seu objetivo principal:

- Survey exploratória: pesquisa primária sobre um dado fenômeno, a qual objetiva adquirir dados para serem utilizados como norteadores de uma pesquisa mais aprofundada. A representatividade da amostra nesse tipo de survey não se faz necessária;

- Survey descritiva: consiste em entender a importância do fenômeno e caracterizá-lo em relação à população, fornecendo base para construção ou aprimoramento de teorias.

- Survey explanatória: demanda requisitos mais detalhados, logo, é a pesquisa que resulta em informações mais robustas e precisas, objetivando o teste de adequação das variáveis relacionadas ao fenômeno, onde a representatividade da amostra é sistemática com propósitos definidos. Além disso, ela deve ser suficiente para a realização de testes estatísticos.

Devido à proposta inicial deste trabalho, escolheu-se a survey exploratória para obtenção de dados, pois não existiam dados de acesso público sobre o assunto em questão, relacionados ao território de Teófilo Otoni, enquanto a pesquisa era realizada.

\section{Representatividade da amostra}

Em pesquisas, define-se população como o número total do público alvo. Para saber o tamanho da amostra da pesquisa é necessário, primeiramente, definir o público. Se a pesquisa se destina a obter a opinião de satisfação dos seus clientes, deve ser realizada com eles.

Para se ter $100 \%$ de certeza em uma pesquisa, é preciso realizá-la com todas as pessoas de uma população. Quando não é viável abordar toda a população, faz-se necessário estimar a quantidade da população, através de uma margem de erro desejada.

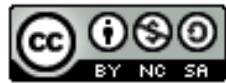


Tabela 1. Margem de erro.

\begin{tabular}{|c|c|c|c|c|}
\hline \multicolumn{5}{|c|}{ Margem de erro desejada } \\
\hline População & $1 \%$ & $3 \%$ & $5 \%$ & $10 \%$ \\
\hline \hline 1.000 & & & 222 & 83 \\
\hline 1.000 & & & 286 & 91 \\
\hline 3.000 & & 1.364 & 353 & 97 \\
\hline 4.000 & & 1.538 & 364 & 98 \\
\hline 5.000 & & 1.667 & 370 & 98 \\
\hline 7.000 & & 1.842 & 378 & 99 \\
\hline 10.000 & 5.000 & 2.000 & 383 & 99 \\
\hline 20.000 & 6.667 & 2.222 & 392 & 100 \\
\hline 50.000 & 8.333 & 2.381 & 397 & 100 \\
\hline 100.000 & 9.091 & 2.439 & 398 & 100 \\
\hline$>100.000$ & 10.000 & 2.500 & 400 & 100 \\
\hline
\end{tabular}

Fonte: Arkin, H., \& Colton, R. R. (1971).

A Tabela 1 mostra a porcentagem da margem de erro e a quantidade de pessoas que é necessário entrevistar para garantir essa margem. Por exemplo, para uma população de 3000 habitantes, fazendo a entrevista com 1364 pessoas, garante-se uma margem de erro de 3\%.

Para obtenção dos dados foram realizadas duas pesquisas. Uma delas, feita por meio de questionário online a respeito da RA, disponibilizado à população via Google Forms e divulgado nas redes sociais em janeiro de 2021, obteve 112 respostas anônimas. A outra, por meio de um levantamento presencial, no centro da cidade, no período de 17 a 25 de maio de 2021, mostrando a RA em prática, contou com 68 entrevistados.

Segundo o Instituto Brasileiro de Geografia e Estatística - IBGE - (2020), Teófilo Otoni possui uma população estimada de 140.937 pessoas. Com base nesses dados, a pesquisa possui margem de erro de $10 \%$, uma vez que foram entrevistadas mais de 100 pessoas.

\section{RESULTADOS E DISCUSSÃO}

Os questionários foram realizados de forma a apresentar, além de questões abertas, a RA na prática, levando para campo o jogo Pokémon Go. O questionário do Google Forms foi divulgado através da rede social WhatsApp, enviado nos grupos da cidade e respondido por um total de 112 pessoas. O levantamento em campo foi realizado na Praça Tiradentes, centro da cidade, onde foram abordadas 68 pessoas de forma aleatória, para que fosse apresentado a elas o jogo, fato que geraria o contato direto com a RA antes da pesquisa.

\section{Análise do Questionário Google Forms}

Analisando os dados da figura 1, depreende-se que, do total questionado, 71,4\% possui idade entre 21 e 40 anos; $15,2 \%$ idade entre 41 e 60 anos; e 13,4\% idade de 10 a 20 anos. 
Figura 1. Gráfico da Idade dos entrevistados.

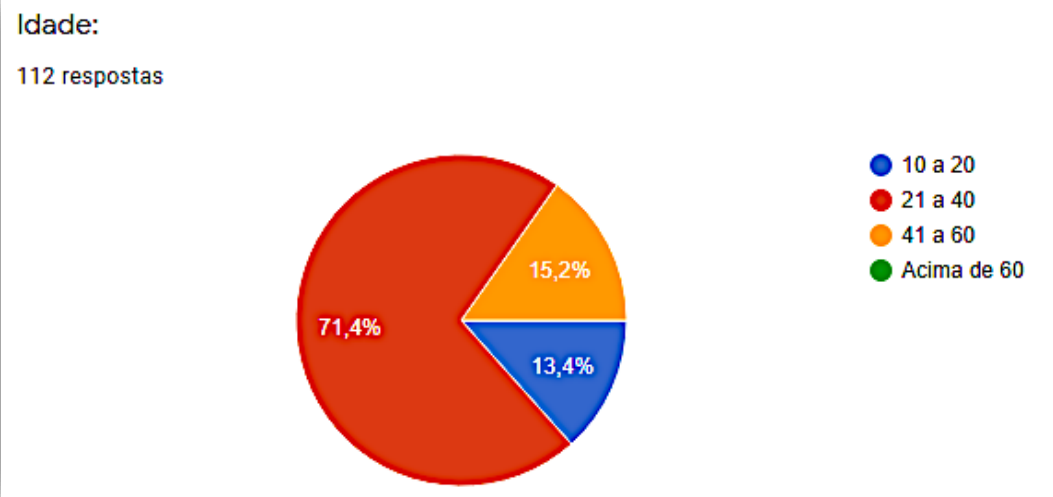

Fonte: Autores (2021).

Observando a Figura 2, somando a escolaridade dos participantes, conclui-se que 60,7\% dos entrevistados estão cursando ou já concluíram o ensino superior. Especificando um pouco mais a Figura 2, tem-se a escolaridade dos participantes, sendo que 33,9\% possuem ensino superior completo; $26,9 \%$ ensino superior incompleto; $25 \%$ ensino médio completo; $10,7 \%$ ensino médio incompleto e $3,6 \%$ outras escolaridades.

Figura 2. Gráfico da Escolaridade dos participantes.

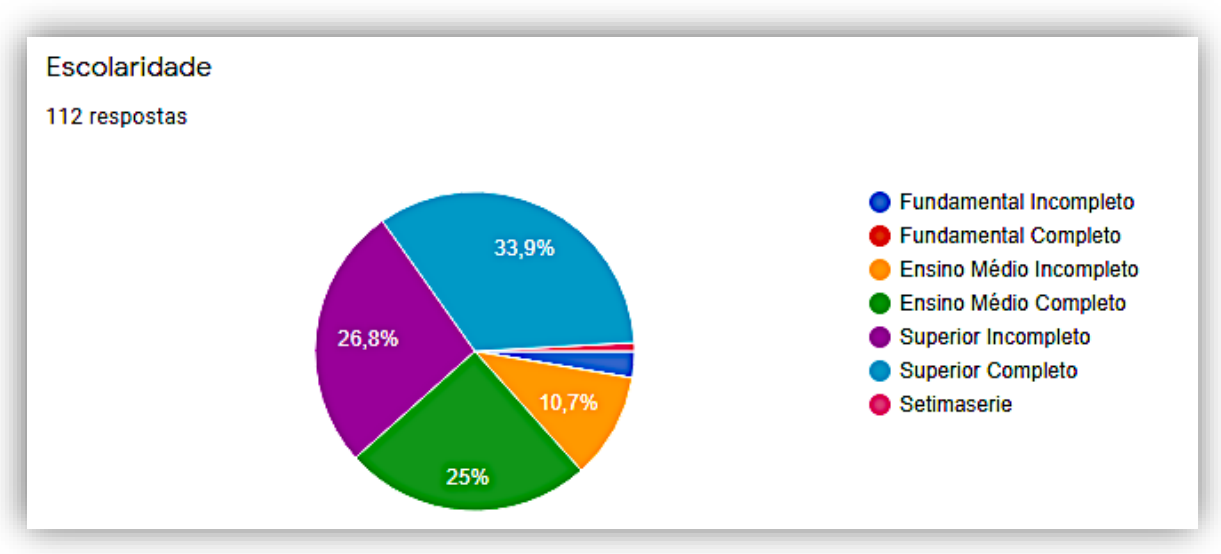

Fonte: Autores (2021).

A Figura 3 mostra o resultado da pergunta "Você sabe o que é realidade aumentada?", em que 64,3\% das respostas foram "não". Percebe-se certa incoerência nas respostas dos sujeitos, haja vista que a pesquisa foi divulgada nas redes sociais, uma delas o Instagram, que faz uso da realidade aumentada, ou seja, a população utiliza a ferramenta e desconhece seu funcionamento. Considerando, também, que a maior parte dos participantes possui ensino superior incompleto ou completo, esperava-se um grau de conhecimento maior sobre o assunto. Observa-se, ainda, que a maior parte dos entrevistados utiliza a RA diariamente, mas não possui conhecimento do que ela seja. 

conhecimento da população teófilo-otonense. Brazilian Journal of Production Engineering, 8(1), 107-117.

Figura 3. Gráfico do Conhecimento sobre a RA.

\section{Você sabe o que é Realidade Aumentada?}

112 respostas

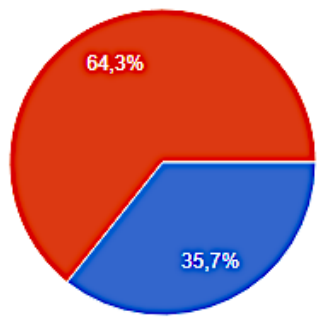

Sim

Fonte: Autores (2021).

Por fim, foi solicitado aos sujeitos que declaram ter conhecimento sobre essa tecnologia que citassem exemplos de RA.

Figura 4. Gráfico dos Exemplos de RA, segundo os participantes.

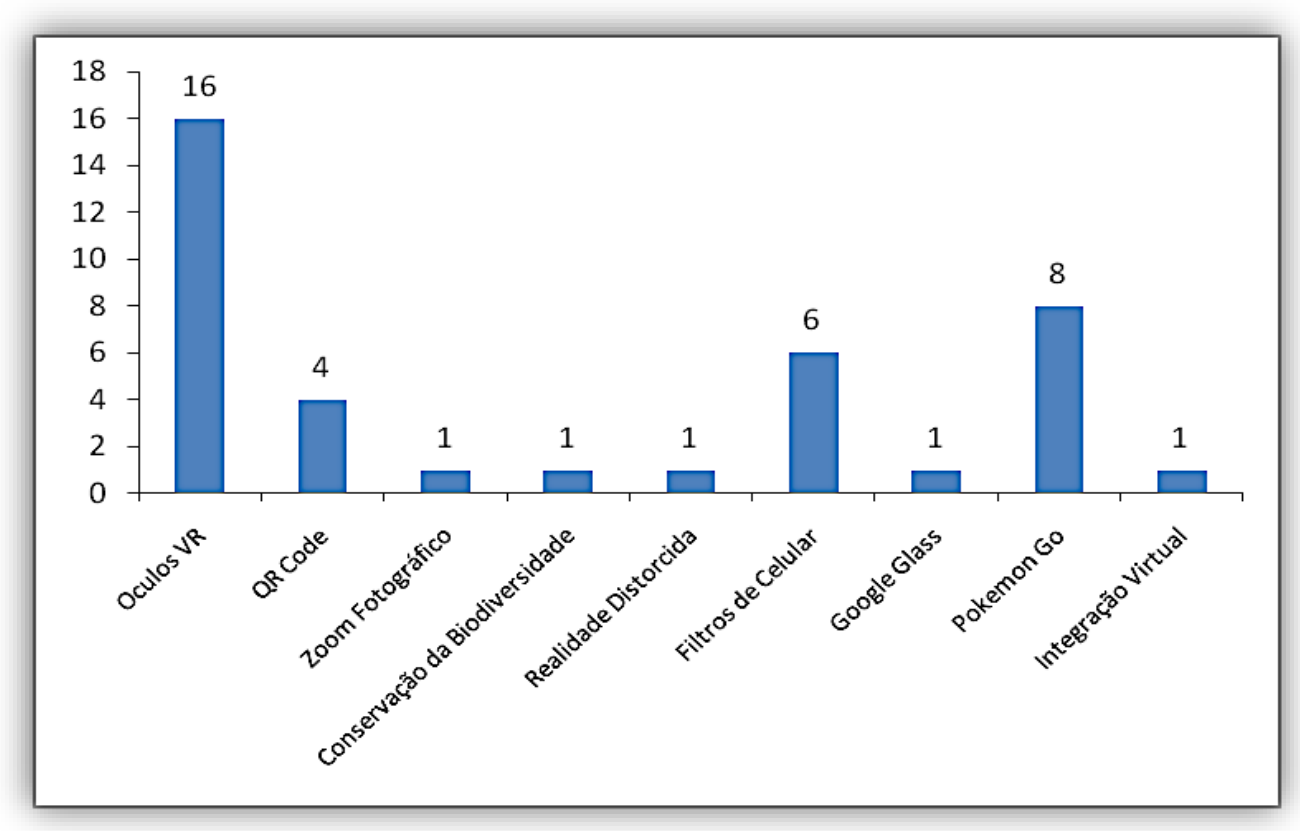

Fonte: Autores (2021).

Dessas respostas, nota-se que as mais comuns foram óculos VR, PokemonGo e filtros de celular. Realizando uma análise mais detalhada, observa-se que das 39 respostas positivas, 19 realmente sabiam do que se tratava; 16 confundem RA com a RV e 4 não conhecem a tecnologia.

Mesmo sendo diferentes, a RA é confundida com a RV, provavelmente pelo fato de a RV ser mais popular e mais antiga no mundo tecnológico. Segundo Kirner (2007) tanto a RA quanto a RV trata de objetos gerados por computadores, mas, no mundo físico, a realidade aumentada está ligada à realidade física, enquanto a realidade virtual refere-se ao sentido de tele presença. Portanto, o fato de ambos serem vistos como "virtuais", ou apenas o fato de a RV ser mais popular e antiga no mundo tecnológico, gera confusão de conceitos na população. 

conhecimento da população teófilo-otonense. Brazilian Journal of Production Engineering, 8(1), 107-117.

Figura 5. Gráfico da Análise dos exemplos de RA.

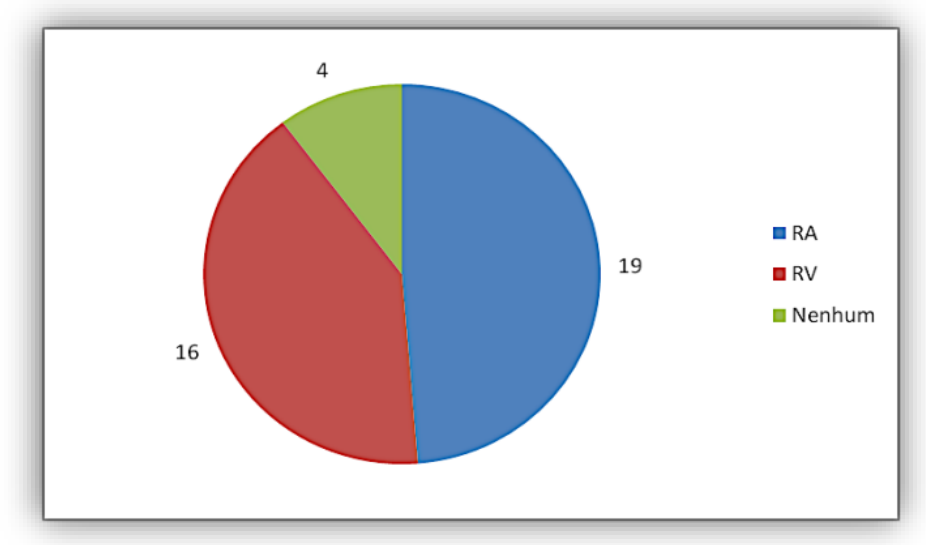

Fonte: Autores (2021).

\section{Análise do Levantamento em Campo}

Analisando os dados da figura 6 , vê-se que, do total questionado, 48,5\% possui idade entre 21 e 40 anos; $42,6 \%$ idade entre 10 e 20 anos; e 8,8\% idade entre 41 e 60 anos.

Figura 6. Gráfico da Idade dos Entrevistados.

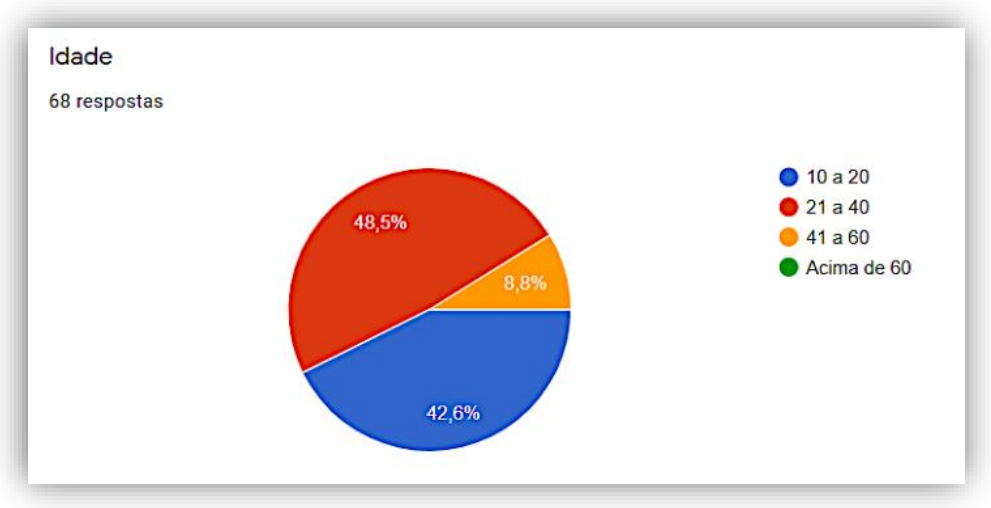

Fonte: Autores (2021).

A Figura 7 aponta a escolaridade dos entrevistados, onde se tem: 30,9\% ensino médio completo; $25 \%$ ensino superior completo; $17,6 \%$ ensino superior incompleto; $14,7 \%$ ensino médio incompleto; e 11,8\% outras escolaridades.

Figura 7. Gráfico do Grau de Escolaridade dos Entrevistados.

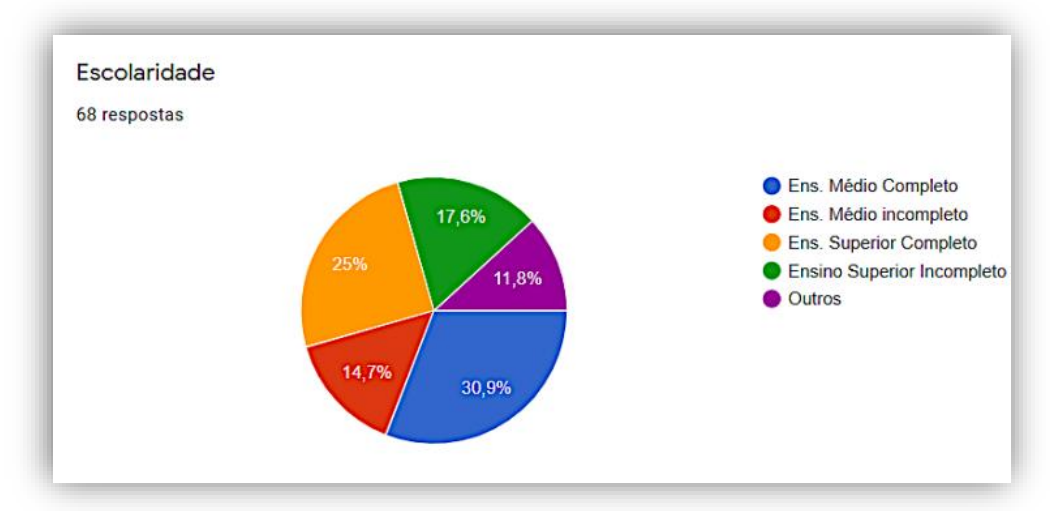

Fonte: Autores (2021). 

conhecimento da população teófilo-otonense. Brazilian Journal of Production Engineering, 8(1), 107-117.

Figura 8. Foto da RA Utilizada no Pokémon Go da Pesquisa.

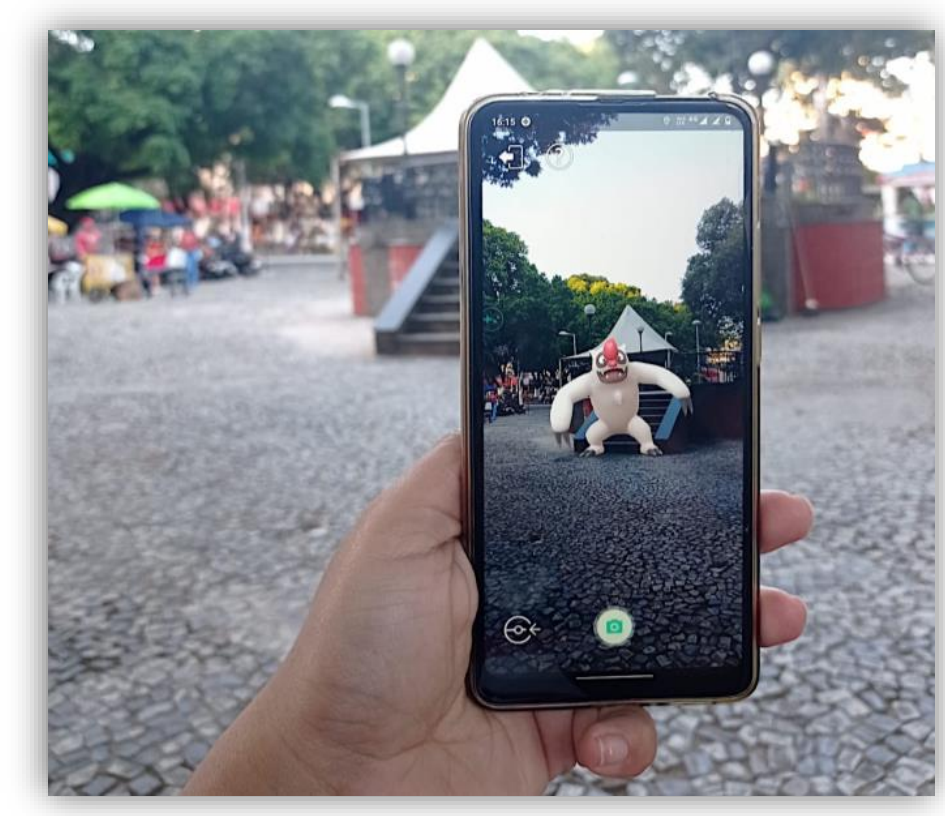

Fonte: Autores (2021).

Na Figura 8, a RA é demonstrada na prática, sendo apresentada aos entrevistados por meio do jogo Pokémon Go. Ao apresentar o Pokémon inserido no ambiente da entrevista, perguntou-se às pessoas se tinham conhecimento de qual tipo de realidade estava sendo retratada. Obtivemos os seguintes resultados, visualizados na Figura 9: 72,1\% disseram RV; 14,7\% RA; $7,4 \%$ outros; e $5,8 \%$ XR.

Figura 9. Gráfico do Resultado do Questionamento.

De acordo com a tecnologia apresentada do jogo Pokémon Go, qual a realidade apresentada:

68 respostas

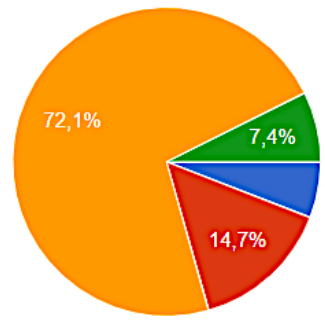

Realidade Estendida

Fonte: Autores (2021).

Percebe-se que, mesmo a RA sendo apresentada na prática aos participantes, o resultado do levantamento de campo foi semelhante à pesquisa on-line, comprovando que a RA é confundida com a RV.

\section{CONSIDERAÇÕES FINAIS}

Expostos todos os resultados, pôde-se obter uma prévia panorâmica do conhecimento da populaçãoda cidade de Teófilo Otonisobre a RA. Percebe-se que parte da população não

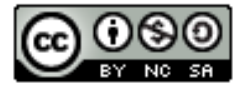


conhece a tecnologia em questão, e os que têm um pouco de informação sobre a mesma confundem-na com a RV, revelando a desinformação dos sujeitos sobre o tema.

Com a falta de conhecimento sobre a RA, a população deixa de usufruir dessa tecnologia, como por exemplo, utilizar os códigos QR na realização de pagamentos ou para obter informações importantes em determinados ambientes. A RA está sendo aplicada nas mais diversas áreas, tais como a educação, a robótica, a medicina entre outras. Sendo assim, é importante que a população conheça sua existência e utilidade. Uma forma da população se inteirar desse assunto éa realização de palestras, exposições em praças públicas, oficinas dentro dos centros acadêmicos abertos à população de forma franca, entre outras atividades.

Como afirma Meira (2021),a possibilidade de utilização da RA em diversos dispositivos a torna fácil de ser aplicada e alcançável a diversos níveis sociais e econômicos. Mesmo sendo mais utilizada na indústria, a sua aplicabilidade no comercio de produtos é bem-vista pelos utilizadores.

Por meio da RA é possível o desenvolvimento de aplicativos para serem utilizados no ecommerce, permitindo que os clientes experimentem de forma virtual o produto que deseja. Com essa possibilidade, a satisfação e o número de compras seria maior, fazendo com que se movimentasse ainda mais o comércio eletrônico e a economia do país. $\mathrm{O}$ e-commerce vem ganhando espaço e se tornando cada vez mais comum. Atualmente o comércio de roupas, eletrônicos, objetos do dia a dia e até mesmo casas e carros são realizados virtualmente, fazendo com que a compra de qualquer bem seja realizada de forma fácil e rápida.

Até o presente momento não foram encontradas pesquisas que abordem as áreas de conhecimento populacional sobre a RA, sendo esse um desafio da pesquisa.Por isso, sugerese que sejam feitas pesquisas mais aprofundadas a respeito da integração da população teófilo-otonense ao "mundo" da Realidade Aumentada, e, quem sabe, a criação de políticas públicas com ênfase na tecnologia.

\section{REFERÊNCIAS BIBLIOGRÁFICAS}

Abreu, D. (2020). Brasil avança quatro posições no Índice Global de Inovação e chega ao $62^{a}$ lugar. Agência CNI de Noticias. Recuperado de https://noticias.portaldaindustria.com.br/noticias/inovacao-e-tecnologia/brasil-avanca-quatroposicoes-no-indice-global-de-inovacao-e-chega-ao-62a-lugar/

Andrion, R. (2019). Realidade Aumentada: você sabe o que é? Olhar Digital. Recuperado de https://olhardigital.com.br/2019/06/29/noticias/realidade-aumentada-voce-sabe-o-que-e/

Arkin, H. \& Colton, R. R. (1971). Tables for statisticians. Barnes and Noble..

Camargo, V. A. X., de. (2010). Realidade Aumentada Interativa: um Estudo de Caso com o Ensino do Movimento Circular. VII Workshop de Realidade Virtual e Aumentada (WRVA'2010). São Paulo.

Cintra, A. [s.d.]. O que é a realidade aumentada e como ela funciona? Post Digital, Recuperado de https://www.postdigital.cc/blog/artigo/o-que-e-realidade-aumentada-e-comoela-funciona

Coifman, A. (2019). Conheça a Realidade Aumentada e suas aplicações. Blog Cronapp. Recuperado de https://blog.cronapp.io/realidade-aumentada-e-suas-aplicacoes/ 
Citação (APA): Dias, D. F. C., Alves, A. F., de S., \& Pereira, L. D., de L. (2022). Análise da realidade aumentada em relação ao conhecimento da população teófilo-otonense. Brazilian Journal of Production Engineering, 8(1), 107-117.

Blog Impacta (2020). Diferenças entre realidade virtual e realidade aumentada! Recuperado de $\quad$ https://www.impacta.com.br/blog/diferencas-entre-realidade-virtual-e-realidadeaumentada/

Instituto Brasileiro de Geografia e Estatística (IBGE). Recuperado de https://www.ibge.gov.br/cidades-e-estados/mg/teofilo-otoni.html

Agência ANSA (2020). Itália realiza $1^{a}$ cirurgia do mundo com realidade aumentada Revista Digital Época Negócios. Recuperado de https://epocanegocios.globo.com/Tecnologia/noticia/2020/02/italia-realiza-1-cirurgia-domundo-com-realidade-aumentada.html

Kirner, C., Tori, R., \& Siscoutto, R. (2006). Fundamentos e Tecnologia de Realidade Virtual e Aumentada. Livro do Pré-Simpósio VIII Symposiumon Virtual Reality. Pará.

Kirner, C. \& Siscoutto, R. (2007). Realidade virtual e aumentada: conceitos, projeto e aplicações. In Livro do IX Symposium on Virtual and Augmented Reality, Petrópolis (RJ), Porto Alegre: SBC (Vol. 28).

Kirner, C. (1996). Apostila do ciclo de palestras de realidade virtual. Atividade do Projeto AVVIC-CNPq (Protem - CC - fase III) - DC/UFSCar, São Carlos, pp. 1-10, Out.

Laura (2020). Realidade Virtual, Aumentada e Estendida: Entenda as diferenças. Revirtua. Recuperado de https://revirtua.com.br/realidade-virtual-aumentada-e-estendida-entenda-asdiferencas/

Lucena, B., de F. (2013). Realidade Aumentada em Celulares: Um estudo sobre a tecnologia e seus potenciais. Tese (Mestrado em Design) - Pontifica Universidade Católica do Rio de Janeiro. Rio de Janeiro, Coleção Digital.

Maciel, I. M. S. (2020). Mapeamento do setor de realidade virtual e realidade aumentada: perspectivas e desafios. I congresso ibero-americano interdisciplinar de economia criativa, 16 a 19 de novembro de 2020, ESPM-Rio, Rio de Janeiro, RJ.

Meira, L., Tori, R., \& Huanca, C. M. O uso da Realidade Aumentada no ensino de Enfermagem.

Mendonça, H. G., de. (2016). E-commerce. Revista Inovação, Projetos e Tecnologias. IPTEC. $4(2)$.

Milgram, P. et al., (1995). Augmented reality: a class of displays on the reality-virtuality continuum. In: Telemanipulator and telepresence technologies. International Society for Optics and Photonics.

Paiola, C. (2019). 4 aplicações da realidade aumentada (VA) na indústria 4.0. it fórum,. Recuperado de https://itforum.com.br/colunas/4-aplicacoes-de-realidade-aumentada-vr-naindustria-4-0/

Equipe TOTVS (2020). Realidade Aumentada: Funcionalidades e Perspectivas. Recuperado de https://www.totvs.com/blog/inovacoes/realidade-aumentada/

Iberdrola, [s.d.]. Realidade Aumentada: O Mundo Real Com Outros Olhos. Recuperado de https://www.iberdrola.com/inovacao/o-que-e-realidade-aumentada

In Club /(2020). Realidade Virtual, Aumentada, Estendida. Qual É A Sua Realidade? Recuperado de http://www.inclublicita.com.br/realidade-virtual-aumentada-estendida-qual-ea-sua-realidade/

Panorama Positivo (2019). Saiba as diferenças entre realidade estendida, virtual $e$ aumentada. Recuperado de https://www.meupositivo.com.br/panoramapositivo/infograficorealidade-estendida-virtual-e-aumentada/ 